\title{
Diversity neither in promises nor whispers only : gender and other factors from the OTRv4 project.
}

\author{
Camille Akmut
}

February 6, 2020

\begin{abstract}
Commits : $>1$. (Three more participants were part of this project and had each 1 commit only.) Brief remarks on select other projects.
\end{abstract}




\begin{tabular}{l|c|r|l|l}
1 & claucece & 975 commits & Sofia Celi & $\mathrm{f}$ \\
\hline 2 & juniorz & 151 commits & Reinaldo de Souza Jr & $\mathrm{m}$ \\
\hline 3 & tcz001 & 91 commits & $?$ & $?$ \\
\hline 4 & rosatolen & 71 commits & Rosalie Tolentino (?) & $\mathrm{f}(?)$ \\
\hline 5 & tdruiva & 65 commits & Tania S & $\mathrm{f}$ \\
\hline 6 & natalieesk & 63 commits & Natalie Eskinazi & $\mathrm{f}$ \\
\hline 7 & yakiradixon & 58 commits & Yakira Dixon & $?$ \\
\hline 8 & iapazmino & 57 commits & Ivan Pazmino & $\mathrm{m}$ \\
\hline 9 & olabini & 32 commits & Ola Bini & $\mathrm{m}$ \\
\hline 10 & chelseakomlo & 24 commits & Chelsea Komlo & $\mathrm{f}$ \\
\hline 11 & annacruz & 20 commits & Anna Cruz & $\mathrm{f}$ \\
\hline 12 & DrWhax & 19 commits & Jurre van Bergen $(?)$ & $\mathrm{m}(?)$ \\
\hline 13 & deniscostadsc & 15 commits & Denis Costa & $\mathrm{m}$ \\
\hline 14 & cobratbq & 11 commits & $?$ & $\mathrm{~m}$
\end{tabular}

Table 1: Diversity in the OTRv4 project

measured 06/02/2020 c. $12.30 \mathrm{pm}$

There are at least 6 women for 14 participants among those who have made more than one recorded contribution.

In that population, we observe also a certain diversity in terms of white and black or non-white participants as well as geographical origins (at least 3 participants were from South America, for instance).

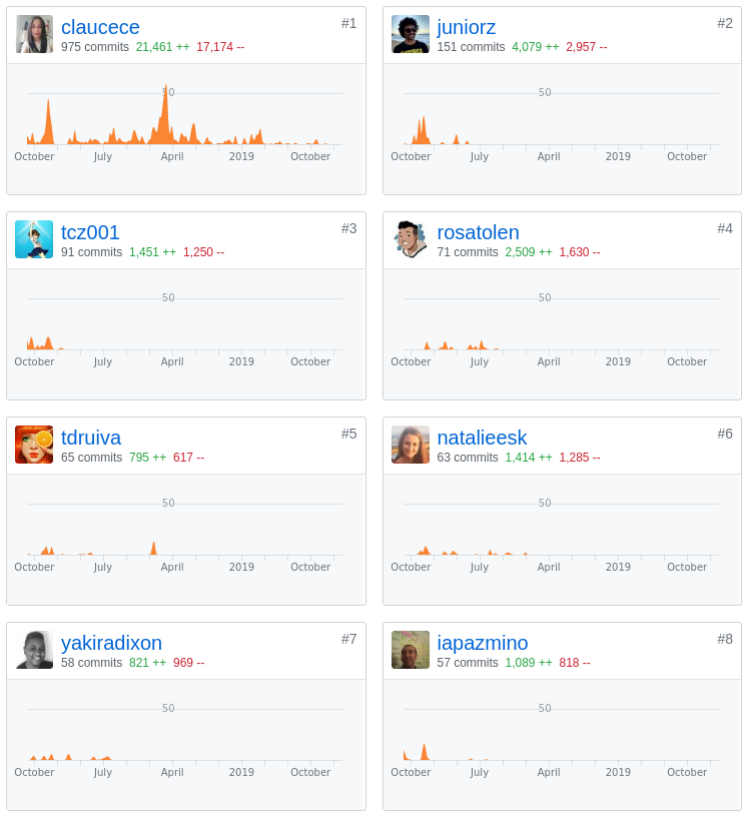




\section{Conclusion}

A series of publications on diversity hereby comes to an end.

Of all projects reviewed so far, this one came closest to what we had pictured as "diverse".

Diversity : a problematic notion, yet it remains that projects such as GNU or the Linux kernel, lead almost entirely by men, and resulting in extraordinarily aberrant statistics such as "100\%" or "99\%" per cent male participants, cannot be said to be diverse by any stretch of the imagination or the human mind.

No matter their grand ideals, and important mission, their structures are reminiscent at best of the 19th century, rather than the 21st.

They mix high technology with bygone all-male college culture and exclusive philosophy clubs.

In choosing one last project, we had considered two others : gnome-shell and GNU guix, among others.

Both were excluded : top contributors to gnome-shell (an important part of GNOME, and with which users interact all the time, whether they know it or not) rapidly appeared to be mostly all male at the time of review.

7 days of contributions to GNU guix were reviewed as well, and either all women of this project had decided to go on vacation together at the same time or there were none here either...

In both cases, this is questionable considering the GNOME project is behind Outreachy, an initiative for the promotion of women, but does not seem to be able to achieve gender diversity in their own project;

while leaders of the GNU guix team - all men - claimed recently to speak for "all users". An absurdity on top of a tragedy : their common statement was in majority if not entirely made up of men... Their logic : incomprehensible.

At the time of writing mesa's top contributor was female, while the Dolphin project's Rachel Bryk remained amongst leading contributors even long after her death, by suicide.

Diversity is certainly a complicated topic, but rarely is a calculator needed. These gentlemen speak in riddles, promises and whispers only :

So discrete, we can neither hear them nor give them any credibility; no more than we can companies doing the same. 


\section{References}

- Miller, Michael. 2015. "'Killed myself. Sorry.': Transgender game developer jumps off bridge..." The Washington Post, 28/04.

- https://github.com/otrv4/otrv4/graphs/contributors

- https://gitlab.freedesktop.org/mesa/mesa/-/graphs/master

- Courtes, Ludovic, Wurmus, Ricardo, Lee, Matt <and some more gentlemen $>$. 2019. "Joint statement on the GNU Project". GNU Guix website. 